\title{
Determination of relationship and frequency of sensorineural hearing loss in previously diagnosed diabetes mellitus and/or hypertensive adults using qualitative semi-structured interviews
}

\author{
Article by Amrit Karmarkar \\ PhD Research Scholar, Texila American University \\ Email:-abkarmarkar@gmail.com
}

\begin{abstract}
Background: Sensorineural hearing loss (SNHL) is most common form of hearing loss, (90\% of hearing loss cases). It was observed that diabetes and hypertension both can cause SNHL through diabetic microangiography and hypertensive retinopathy in ear respectively. Considering prevalence of diabetes and hypertension in India, particularly in state of Maharashtra and their association with sensorineural hearing loss, it was decided to evaluate relationship and frequency of SNHL in adult population of Mumbai metropolitan region.

Methodology: The aim of study was to investigate relationship and frequency of SNHL due to previously diagnosed diabetes mellitus and/or hypertension in adult population of Mumbai metropolitan region. In present study, protocol was designed for semi-structured qualitative interviews of physicians to determine relationship and prevalence of SNHL. In total 25 respondents were replied to semi-structured interviews.

Results: Semi-structured interviews of physicians and audiologists revealed that there exists close association with duration and control of diabetes and/or hypertension. In semi-structured interviews it was found that out of all SNHL patients, there were $71.43 \%$ cases of diabetes; 39.29\% cases of hypertension; and $53.57 \%$ of both diabetes and hypertension. Severity of hearing loss due to diabetes and/or hypertension was found to be moderate with more prevalence in age group of 50-60 years.

Conclusion: It was found that sensorineural hearing loss occurs due to diabetes and/or hypertension. Better control of these conditions at early stage might prevent causation or progression of sensorineural hearing loss. There is need to create awareness amongst public about hearing loss and utility of hearing aids.
\end{abstract}

Keywords: Sensorineural hearing loss, diabetes, hypertension, semi-structured interviews.

\section{Introduction}

Sensorineural hearing loss is most common form of hearing impairment amongst human population. It is accounted to be $90 \%$ of all hearing disorders (Latoche, Neely \& Noben-Trauth, 2011). SNHL, sometimes referred as "nerve deafness" which results due to dysfunction in inner ear i.e. cochlea or cranial nerve VIII (auditory nerve) or due to any dysfunction in central nervous system processing (Bhattacharyya, \& Thaj 2010). As per updated report of WHO in 2013, prevalence of hearing loss increases as per age with maximum incidence above age of 65.

This is estimated to be $50 \%$ in South Asia and 18\% in high income region (Duthey 2013, Shield 2006). Report from Shield (2006) states that 1 in 7 people in UK or around 9 million people are suffering from hearing impairment. Statistics in India suggests that one in twelve persons have hearing loss (Project Deaf India, 2013).

Recent studies have shown that diabetes and hypertension have close relationship to cause sensorineural hearing loss. Since 1961, studies are underway to investigate relationship of diabetes and hearing loss. Microvascular complications occur in ear 
South American Journal of Clinical Research

Special Edition 2016

due to diabetes leading to thickening of basement membrane of capillaries of inner ear. Diabetes causes diabetic microangiopathy leading to thickening of vascular membranes and vascular endothelium of ear. Reduced flow due to narrowing of vasculature leads to degeneration of VIII cranial nerve (Makishima \& Tanaka 1971, Taylor \& Irwin 1978, Costa 1967, Smith, Raynor, Prazma, Buenting \& Pillsbury 1995). In study by Makishima et al. (1971), it was found that in diabetic individuals, demyelination of auditory nerve occurs due to degeneration of myelin sheath. Apart from this; alterations in axon, atrophy in spinal ganglion leading to cellular loss in cochlear basal and middle turns and loss of nerve fibres in spiral lamina occurs. These collectively lead to sensorineural hearing loss. The cross sectional clinical trial conducted by Diniz and Guida (2009) wherein worse audiometric thresholds were seen amongst diabetes mellitus patients. Increased levels of serum creatinine due to poor control of blood sugar level in diabetic individuals causes diabetic microangiopathy leading to sensorineural hearing loss (Kakarlapudi, Sayer \& Staecker 2003). Age at onset and duration of diabetes is also associated with SNHL (Mozaffari, Tajik, Ariaei, Ali-Ehayaii \& Behnam 2010). Study by Nishio et al. (2012) suggests that polymorphism of complement factor $\mathrm{H}(\mathrm{CFHY} 402 \mathrm{H})$ in diabetic individual make them more susceptible to sudden sensorineural hearing loss.

As per criteria set by NICE guideline, elevated blood pressure in arteries above 140 (systolic) and 90 (diastolic) $\mathrm{mm} \mathrm{Hg}$ is regarded as hypertension (NICE Clinical Guideline 127). High blood pressure was also found to be associated with SNHL (Mondell \& Lopes 2009, Marchiori, Filbo \& Mastuo 2006). In 1982, McCormick et al. (1982) investigated relationship of hypertension in rats with reduced cochlear potentials. Findings indicated that hypertension is important risk factor in age related hearing loss. Another study by Sui et al (2003) also reported similar findings in Wistar rats. Report from US National Health and Nutrition Examination Survey from 1999 to 2002 suggest that cardiovascular risk factors (hypertension, smoking, and diabetes) are responsible for hearing loss (Agrawal, Platz, \& Niparko 2009).

Hypertension may lead to structural changes in blood supply of inner ear or may cause inner ear haemorrhage leading to sudden or progressive hearing loss. Underlying pathological mechanisms might be increased blood viscosity, reduction in capillary blood flow, reduction in oxygen transport and hypoxia, changes in ionic cell potentials. These cumulatively lead to hypertensive retinopathy (i.e. damage in end organ blood vessels). This in turn results in problems in hearing capacity (Marchiori, Filbo \& Mastuo 2006, Tan, Rahmat, Prepageran, Fauzi, Noran \& Raman 2009). Other two studies by Esparza et al (2007) and Wallhagen et al (1997) also indicate relationship of hypertension and sensorineural hearing loss.

Most of times, hypertension is associated with diabetes. Findings from Screening India's Twin Epidemic (SITE) study suggest that one in five of Indian adults and one in three in state of Maharashtra suffer from both diabetes and hypertension (Joshi, Vadivale, Dalal \& Das 2011, Viswanathan, Seedat \& Pradeepa 2013). In UK, 3 people out of 10 who have type 1 diabetes and 8 people out of 10 with type 2 diabetes have hypertension (Patient.co.uk).

Individual studies to determine association of SNHL with diabetes or hypertension were done earlier. However, only one case control study from Brazil was conducted in order to determine association of both diabetes and hypertension. However, this study examines only idiopathic sudden SNHL and no other forms of SNHL were considered (Nagaoka, Anjos, Takata, Chaim, Barros \& Penido 2010). No study has been conducted considering these conditions and their association with bilateral, progressive or irreversible SNHL. Considering high prevalence of diabetes and hypertension and hearing loss in India, it becomes essential to perform this study to determine association or relationship and frequency of SNHL which occurs due to diabetes and/or hypertension. 


\section{Methodology}

To get detailed insight on one disorder, (i.e. sensorineural hearing loss); study was designed to explore three different perspectives wherein opinion of physicians and audiologists; adult child or family member; and common public can be evaluated.

Semi-structured interviews or qualitative interviews provide detailed information about the topic. As it basically focuses on opinions, results or responses of interviewees might be controversial. However, aim of study remains to stimulate reflection and exploration of information about the disease condition (Davies 2007). Purpose of choosing semi-structured interviews is their value in picking up unique findings of physicians that they have acquired over years of experience as well as some common experiences. These things cannot be explored from conventional questionnaire based surveys. Before preparation of protocol for semi-structured interviews, pre-pilot or exploratory study and pilot study were carried out.

\section{Pre-pilot study}

Pre-pilot study serves as thought clarifying stage for qualitative research (Davies 2007).This involved conversation with one female audiologist with 22 years experience. This conversation serves as essential step in design of questionnaire for protocol of semi-structured interviews.

\section{Preparation of draft of protocol for semi-structured interviews}

After pre-pilot study, relevant literature search was done and draft protocol of semistructured interview was written. Most questions were open ended in order to get more information from physicians and audiologists. Probes were provided so as to obtain detailed view of physicians and audiologists. Protocol contained outline such as introduction, ground rules, brief information, and 24 open ended questions. This protocol was then subjected to pilot study.

\section{Pilot study}

Draft of protocol of semi-structured interviews was sent to Dr.Prof. Phil Warner, Cranfield University, UK and discussed with one MD; two MBBS; one MS (ENT) and one audiologist.

\section{Finalisation of protocol for semi-structured interviews}

Changes suggested by all of them were made and protocol was finalised.

\section{Study design}

This was qualitative research using semi-structured interviews of physicians and audiologists.

Study setting: Study was carried out in private clinics, hospitals, Govt. hospitals of Mumbai metropolitan region.

Study population and sample size: Study involved semi-structured interviews of physicians and audiologists with following qualifications.

Physicians

- MBBS (Bachelor of medicine and bachelor of surgery)

- MD (Doctor of medicine) (Medicine) (Diabetologist and Cardiologists)

- MS (ENT) (Master of surgery in Ear, Nose and Throat)

- Audiologists

- $\quad$ BSc /M Sc AST (Bachelor of Science in Audiology and Speech Therapy)

Exclusion criteria: Physicians practising alternative systems of medicine such as Ayurveda, Siddha, Unanai, Naturopathy, Yoga, Acupuncture, Acupressure, physiotherapy and Homeopathy were excluded from study. 
South American Journal of Clinical Research

Special Edition 2016

Potential participants were identified by using local healthcare professionals' directory (Arogyadeep 2013). They were contacted by using telephone or face to face contacts and appointments were fixed based on mutually agreed suitable time. Participants were informed about study conduction and confidentiality.

Sample size: Total number of participants targeted was calculated from pilot study. Number of professionals targeted was 49.

Study duration: Study was carried out from 08 October 2013 to 01 December 2013.

Recording of semi-structured interviews: Responses of semi-structured interviews of physicians and audiologists were recorded using Sony Walkman NWZ-B163F. All participants were informed prior to recording the interview.

Record keeping and confidentiality: All participants were informed that all records will be kept in electronic mp3 file format on Lenovo B490 laptop in secure folder. Destruction of notes and audio tapes will be done after 5 years of completion of this study.

Ethical considerations: As this is non interventional study of physicians no ethical approval was obtained.

\section{Data collection and analysis}

While taking interview, written notes were taken so as to identify key points in interview. Recording of interview audio tapes were heard and transcribed into notes. This data was categorised so as to convert it into quantitative data and this data was represented graphically in form of bar graphs. Wherever applicable, percentage analysis was done. Data analysis and representation was done using Microsoft Excel 2007.

\section{Results}

Study was designed in order to study three different perspectives such as physicians, family member or adult children and awareness amongst public. Recordings of semi-structured qualitative interviews were transcribed. Along with this, data from study note book was referred.

\section{Respondent's pattern}

In total, 49 physicians and audiologists were approached for semi-structured qualitative interviews. It was found that professionals declined to respond as many of them were not aware about method of semi-structured interviews; they had lack of time; or they were not comfortable with recording of interview session. Out of 49 professionals approached, only 25 physicians and audiologists responded to the invitation. Therefore, response rate observed was 51.02 percent. Physicians approached were qualified (MBBS, MD (medicine), MS (ENT), audiologists) and were having different experience in their profession (0 to 50 years). In total, 9 MBBS, 5 MD (medicine), 6 MS (ENT), 5 audiologists gave their response. More experienced professionals have accurate diagnosis and can provide better treatment. Audiologists look after maximum cases (35) of SNHL per month than other physicians MS (ENT) (28), MD (medicine) (16), MBBS (7).

\section{Investigation of Frequencies of conditions}

Percentage of patients that have both diabetes and SNHL: Questionnaire was focused to determine of percentage of patients of SNHL which have diabetes. This may be estimate of co-existence of these conditions. While interviews, different physicians and audiologists told different figures about co-existence of diabetes with SNHL. Therefore, mean values were determined as per qualifications of professionals and percentages were determined. Out of total percentage of patients of SNHL per month, Figure 1 represents percentage of patients with diabetes and SNHL. Figure 1 showed 
that MS (ENT) specialists have large number of patients (71.43\%) of diabetes and SNHL.

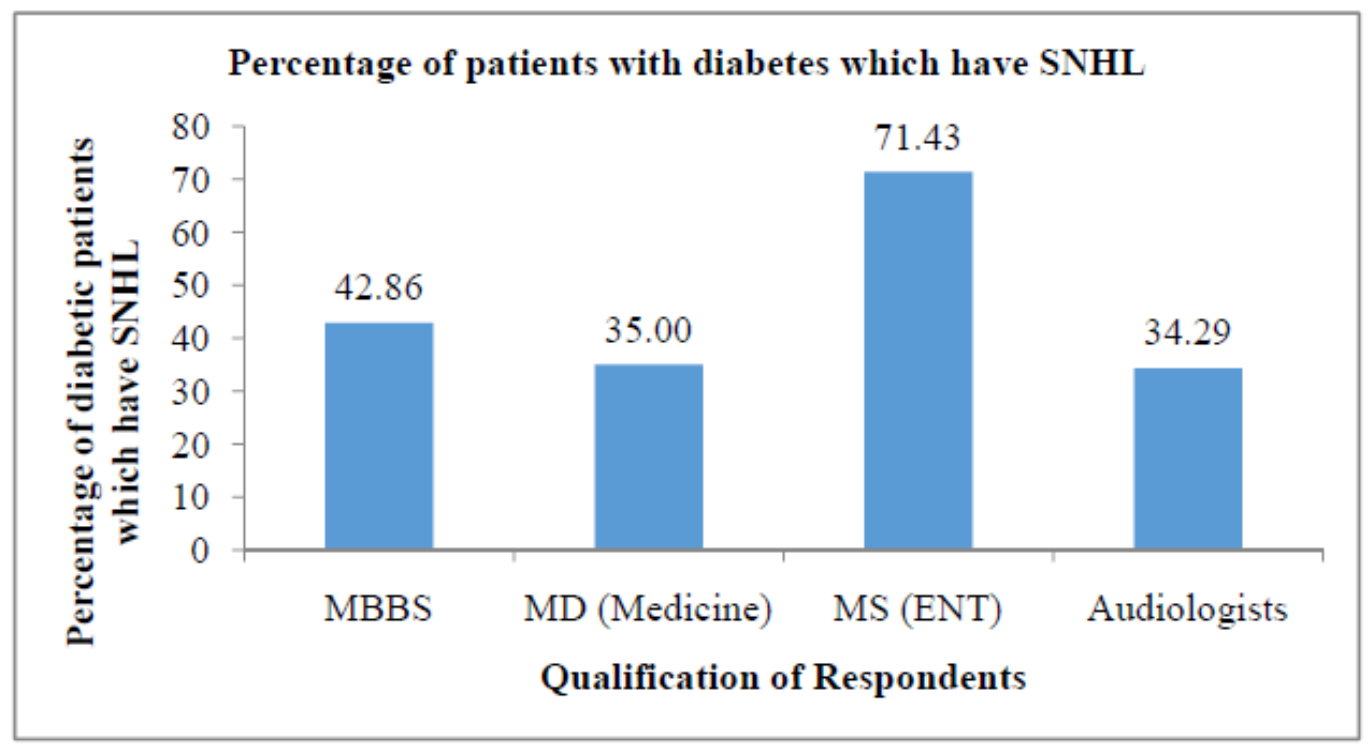

Figure 1: Percentage of patients with diabetes which have sensorineural hearing loss

Percentage of patients of hypertension which have sensorineural hearing loss: Physicians were asked to estimate number of patients of hypertension out of total number of SNHL patients. Therefore, each response was collected and mean value was determined as per their qualifications and percentage was calculated. Figure 2 shows number of patients of hypertension which have SNHL. Figure 2 suggests that there is small difference in number of patients with hypertension and SNHL in every professional (percentage ranges from 34.29 to $42.86 \%$ ).

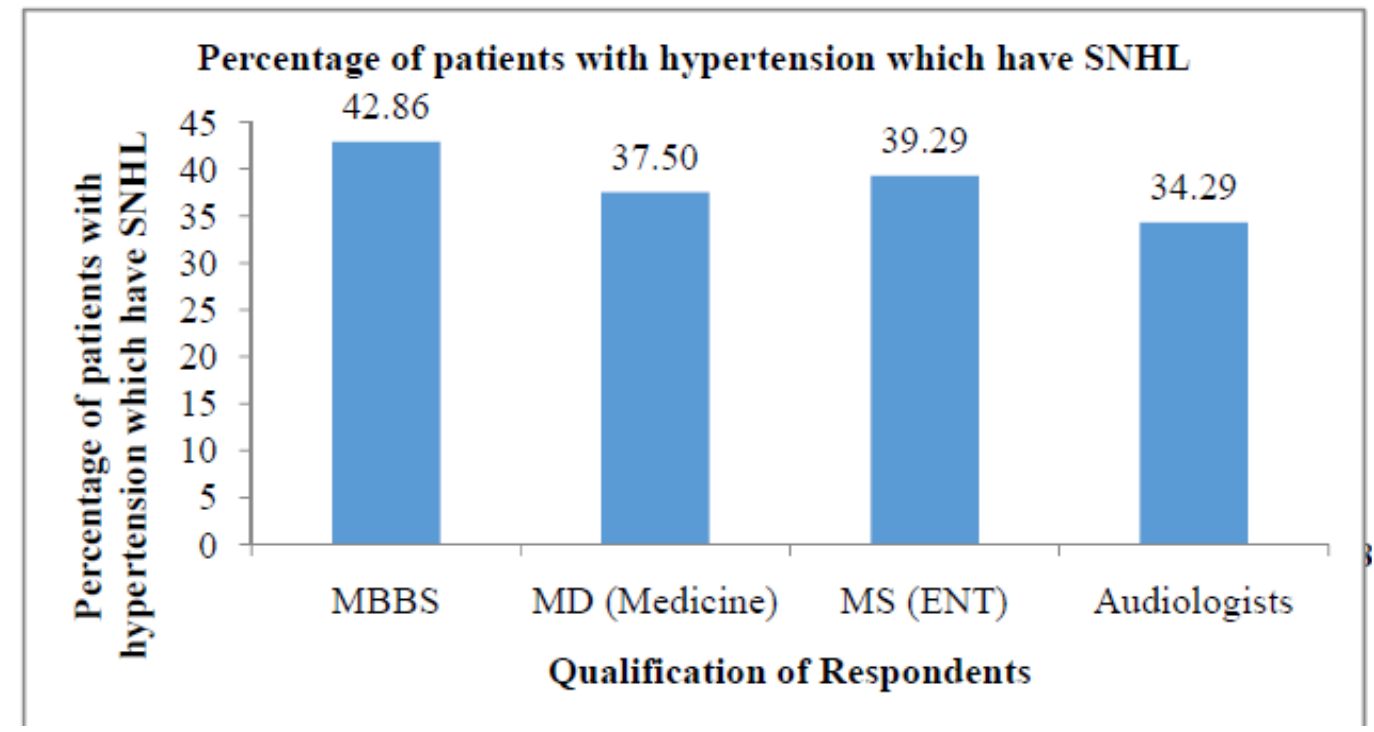

Figure 2: Percentage of patients with hypertension which have sensorineural hearing loss 
South American Journal of Clinical Research

Special Edition 2016

Percentage of patients with both diabetes and hypertension along with SNHL: Diabetes and hypertension alone can cause SNHL. In state of Maharashtra, statistics of SITE study suggests that one in three suffer from both diseases. Therefore, it becomes essential to determine percentage of patients which have both diseases along with SNHL. Figure 3 shows that number of patients with both above conditions out of total percentage of SNHL patients per month. It suggests that ENT specialists observe around $53.57 \%$ of cases and MBBS (General practitioners) observe $42.86 \%$ of cases which is more than MD (Medicine) (31.25\%). The reason behind second rank of MBBS practitioners is that they are referred as family physicians by most of population who manages treatment of both diabetes and hypertension.

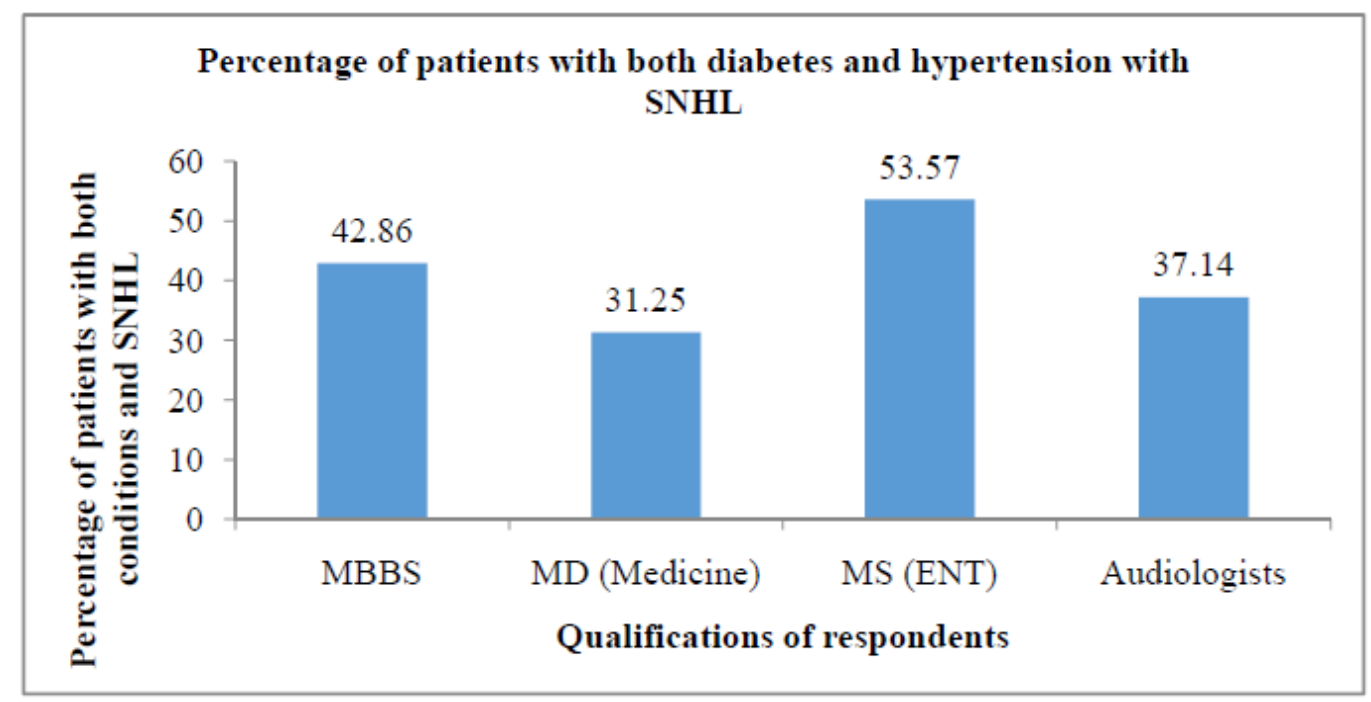

Figure 3: Percentage of patients with both diabetes and hypertension with SNHL patient factors Questions were asked during interview to evaluate duration of occurrence (in time period) of SNHL, age of patients, gender, job description, family history of patient etc.

Physicians gave different responses ranging from 7 years to 10 years for occurrence of SNHL. Mean values as per qualifications of physicians were calculated. This indicates that MBBS, MD (Medicine) and Audiologists suggest duration of occurrence of SNHL as 8 years whereas MS (ENT) suggest it to be 9 years. 52\% of physicians said that patients in age group 50-60, SNHL is observed mostly. This is further supported by following two responses:

A female audiologist with 25 years experience stated following:

"Generally SNHL is observed in age of 55 to 60 years and above. However, it has been observed from case histories, that when patient has diabetes particularly, this age group is around 50 to 60 or sometimes less than 50 also. This can be also true in case of hypertension"

According to male MBBS physician 35 years of experience,

"I think if patient is diagnosed with both diabetes and hypertension, chances of hearing loss increase. This might lower age of occurrence of sensorineural hearing loss. Hence, probably age of SNHL in both cases may be 50-60."

Around 56\% of physicians said, there was no difference in gender to cause SNHL. However, $40 \%$ of physicians said that males were more prone to have SNHL.

Female audiologist with 22 years experience added the fact,

"Although males are more prone to have SNHL, prevalence in females is also higher. But in India, many females are housewives or homemakers. They neglect fact of hearing loss and even working women do not agree to wear hearing aid due to 
issue of aesthetics. So there lies lot of social stigma when prevalence is compared in males and females."

A mixed opinion was received when physicians and audiologists were asked about relation of job description and SNHL. Forty four percent of physicians and audiologists said that factory workers are more prone whereas same percentage of physicians and audiologists said that there is no relation or discrimination due to job description or working style of patient.

However, female audiologist with 25 years of experience whose clinic is near to railway station area said,

"As my clinic is near to railway station, I see many cases of hearing loss. Particularly workers in railway factory or workshop that have diabetes and hypertension are more prone to have SNHL at age of 50-60."

Male MS (ENT) surgeon with 7 years of experience said,

"Factory workers are more prone to have SNHL. However, this depends on location of clinic. As my clinic is situated near to industrial area, I get more number of patients who are factory workers. But this may not be the case of every physician or audiologist."

\section{Physician's recommendations about hearing loss}

MBBS physicians, Audiologists and MD (Medicine) refer to MS (ENT) specialist for detailed examination of sensorineural hearing loss (SNHL). About 76\% physicians observed cases having SNHL in both ears and about $84 \%$ physicians said patient have family history about close relationship with causation of SNHL. Physicians were asked whether they recommend audiometric tests for patients of diabetes and/or hypertension. Results showed that half of respondents said they recommend and half said they do not recommend audiometric tests to patients. About $48 \%$ physicians recommend conduction of audiometric tests after 7-10 years duration of both diabetes and hypertension. Other physicians mostly MBBS and MD (Medicine) did not answer this question. In patients with diabetes and/or hypertension, frequency of audiometric tests is once in year, recommended by $68 \%$ respondents while remaining have not answered this question. Non respondents were majorly MBBS and MD (Medicine) physicians. While performing audiometric tests, all MS (ENT) and audiologists ask their patients about presence of hypertension and/ or diabetes. Different computerised tests such as automated otoacoustic emissions (AOAE) test and automated auditory brainstem response (AABR) are available, but in India, pure tone audiometry and impedance test were used. Pure tone audiometry was used by $72 \%$ of respondents and both pure tone and impedance tests were used by $28 \%$ of respondents.

Inner ear pathology analysis were recommended by only $20 \%$ professionals only if advised by ENT surgeon. Seventy two percent of physicians and audiologists do not refer the patient to have inner ear pathology analysis.

\section{Hearing related conditions}

Degree or severity of condition is important aspect for initiation of treatment or therapy. According to $76 \%$ of respondents, moderate sensorineural hearing loss occurs due to diabetes and/or hypertension. Some physicians suggest use of hearing aid for SNHL. Hearing aids are of different types such as behind the ear (BTE), in the canal (ITC), in the ear (ITE), receiver in canal (RIC), completely in canal (CIC), etc. Even they are available in digital and analog modes. Many respondents have different opinions regarding hearing aids. $60 \%$ physicians recommend BTE type of hearing aid while remaining refer other types almost with equal proportion. Some of the prominent ones are given below.

One female audiologist with 22 years of experience said, 
South American Journal of Clinical Research

Special Edition 2016

"Generally when hearing loss is detected, I provide detailed information about different types and utility of hearing aids. However, choice of hearing aid depends totally on economical condition of patient. Many times, working women feel awkward while wearing behind the ear type, so they prefer to choose completely in canal type. However, this again depends on severity of sensorineural hearing loss.”

One male ENT surgeon with 15 years of experience added,

"Of course pocket of patient i.e. economical condition matters while choice of hearing aid. But in severe cases we don't advice them to have completely in canal type of hearing aid. This creates problematic situation when patient wishes that his/her hearing aid should not be noticed by people but we cannot provide him that type considering his/her severity of sensorineural hearing loss."

One male audiologist with 18 years of experience said,

"Choice of hearing aid is totally dependent on patient. But in India, patients prefer behind the ear (BTE) type due to its low cost. But younger population with 40-50 age groups prefer completely in canal (CIC) type of invisible kind of hearing aids. In very old people with dexterity problems of age above 60, I refer them pocket model or behind the ear. But in total in India, very few patients buy digital hearing aid due to its high cost and need of programming."

Tinnitus is presence of hissing, roaring, ringing or whooshing sound in one or both ears of patient. Tinnitus is most commonly associated with sensorineural hearing loss $^{61}$.

Regarding the relation of tinnitus and SNHL, one male ENT surgeon with 29 years of experience said,

"In SNHL due to diabetes and/or hypertension, nerve and/or hair cell damage of inner ear occurs. In $80 \%$ of such cases subjective tinnitus occurs. This form of tinnitus makes patient difficult to concentrate and sometimes anxious. Therefore, this can be treated by using antidepressant or anti-anxiety drugs or simply using stress management or music therapy."

Out of all respondents, 68\% reported occurrence of tinnitus due to both diabetes and hypertension whereas, $16 \%$ reported occurrence due to diabetes only.

In literature, it was seen that there is a close relation between vertigo and SNHL. Vertigois form of dizziness that occurs due to dysfunction of vestibular system. All respondents agreed this fact that in SNHL due to both diseases diabetes and/or hypertension chances of vertigo increase.

\section{Diabetes, SNHL, hypertension}

Question was asked to professionals regarding preference for treatment whether drug treatment or hearing aid or combination of these two. Respondents showed mixed opinion regarding this. Fifty two percent prefer drug therapy and $40 \%$ refer to hearing aid only and $8 \%$ refer to both of these.

However, one male ENT surgeon with 7 years of experience added,

"Although allopathic drugs are available for treatment, I prefer to use herbal remedies such as Gingko biloba, Ginseng, etc. along with methylcobalamin. In case of severe or profound sensorineural hearing loss I advice patient to have cochlear implant. But this totally depends on economical condition of patient."

Literature have shown, hypertension causes sensorineural hearing loss in patients. Forty percent of respondents said that 160 systolic and 110 diastolic mmHg levels of blood pressure can cause sensorineural hearing loss. Graphically different levels are represented in Figure 4. 


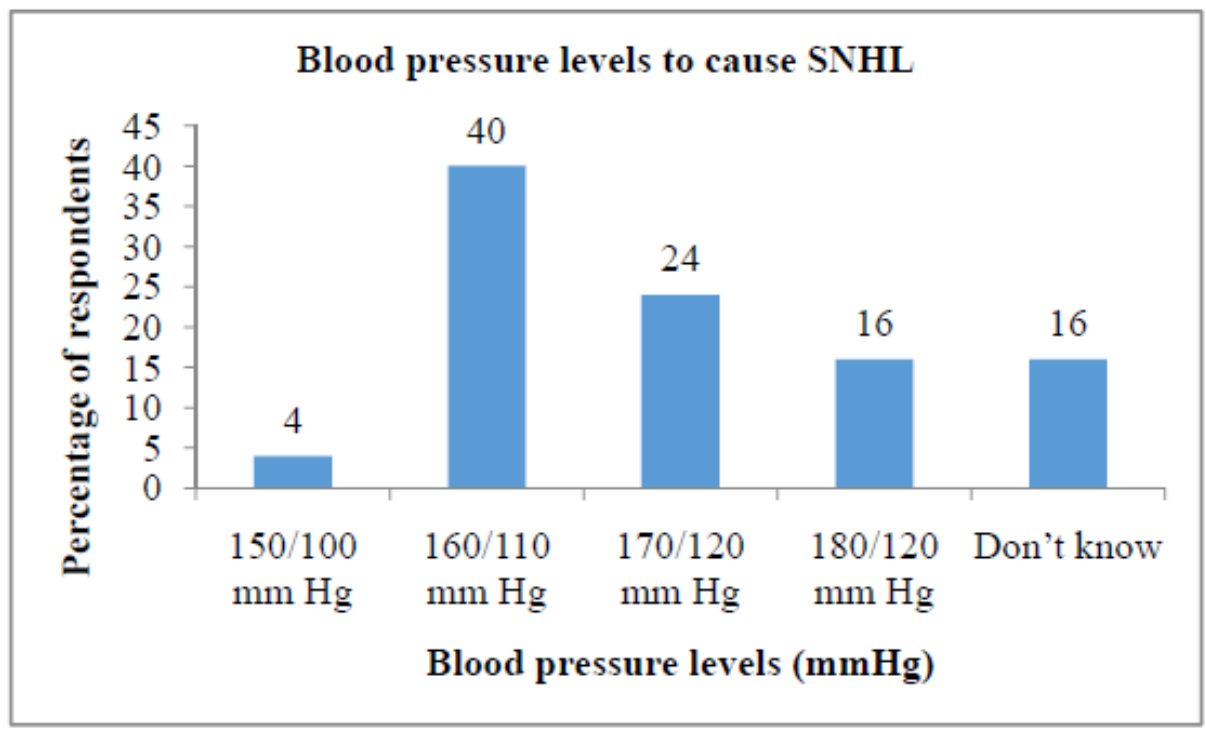

Figure 4: Blood pressure levels to cause SNHL

Duration of hypertension in patients to cause SNHL was asked to physicians and audiologists. Interestingly, half of the professionals (52\%) did not answer question whereas $24 \%$ professionals said it to be 8 years. Figure 5 shows results.

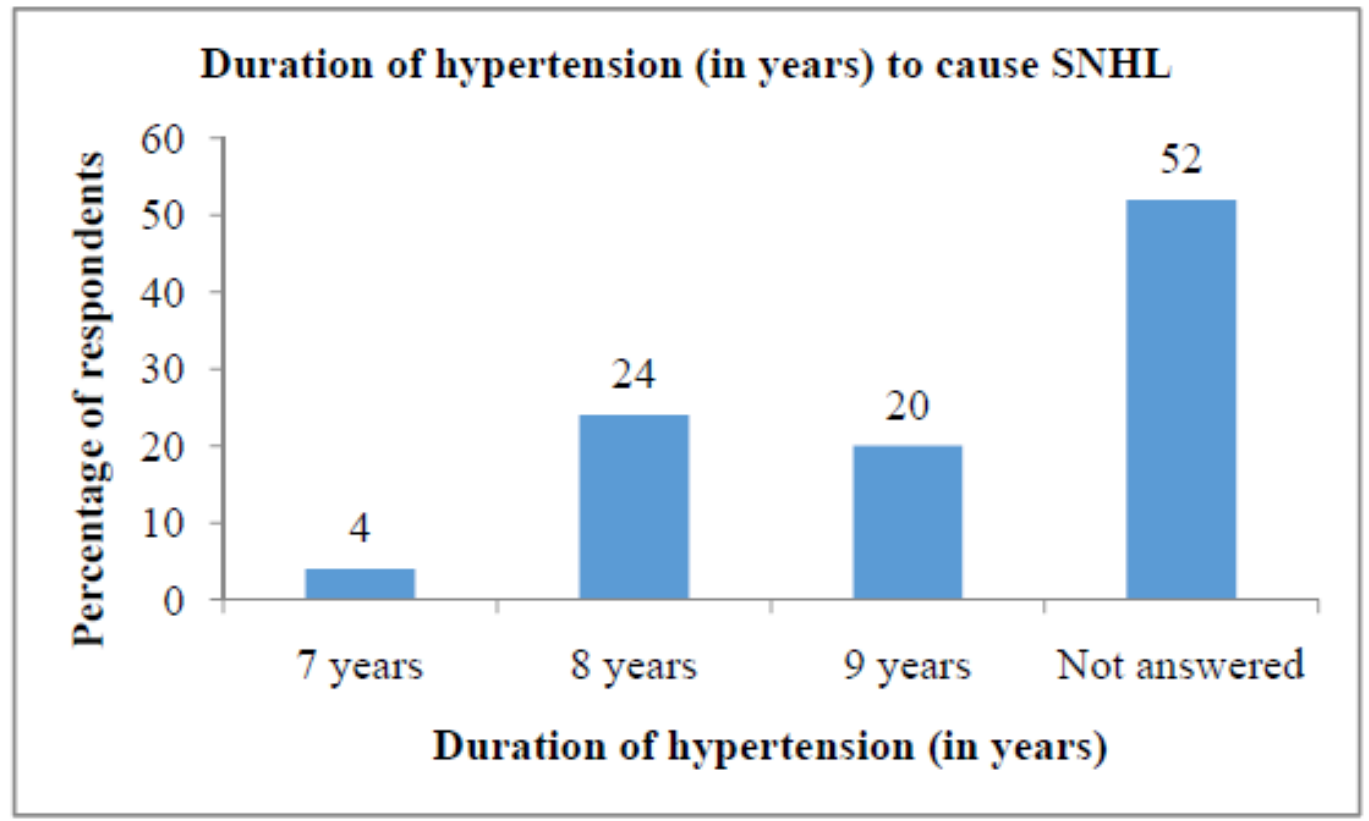

Figure 5: Duration of hypertension to cause SNHL

One male ENT surgeon with 30 years of experience said,

"Along with duration of diabetes and/or hypertension, management of these conditions is essential. As SNHL gets worse over the years if underlying cause is untreated"

Question was asked about if there is any impact of antihypertensive drugs on causation of SNHL. All physicians and professionals did not have knowledge about effect of antihypertensive drugs to cause hearing loss.

Like hypertension, diabetes is also one of the cause for SNHL. Different responses were obtained when professional were asked about blood glucose levels that can 
South American Journal of Clinical Research Special Edition 2016

cause SNHL. These responses were categorised into groups and mean levels were considered. 32\% of physicians think that glucose levels of 200/350 (fasting/postprandial) can cause SNHL. This is represented in figure 6.

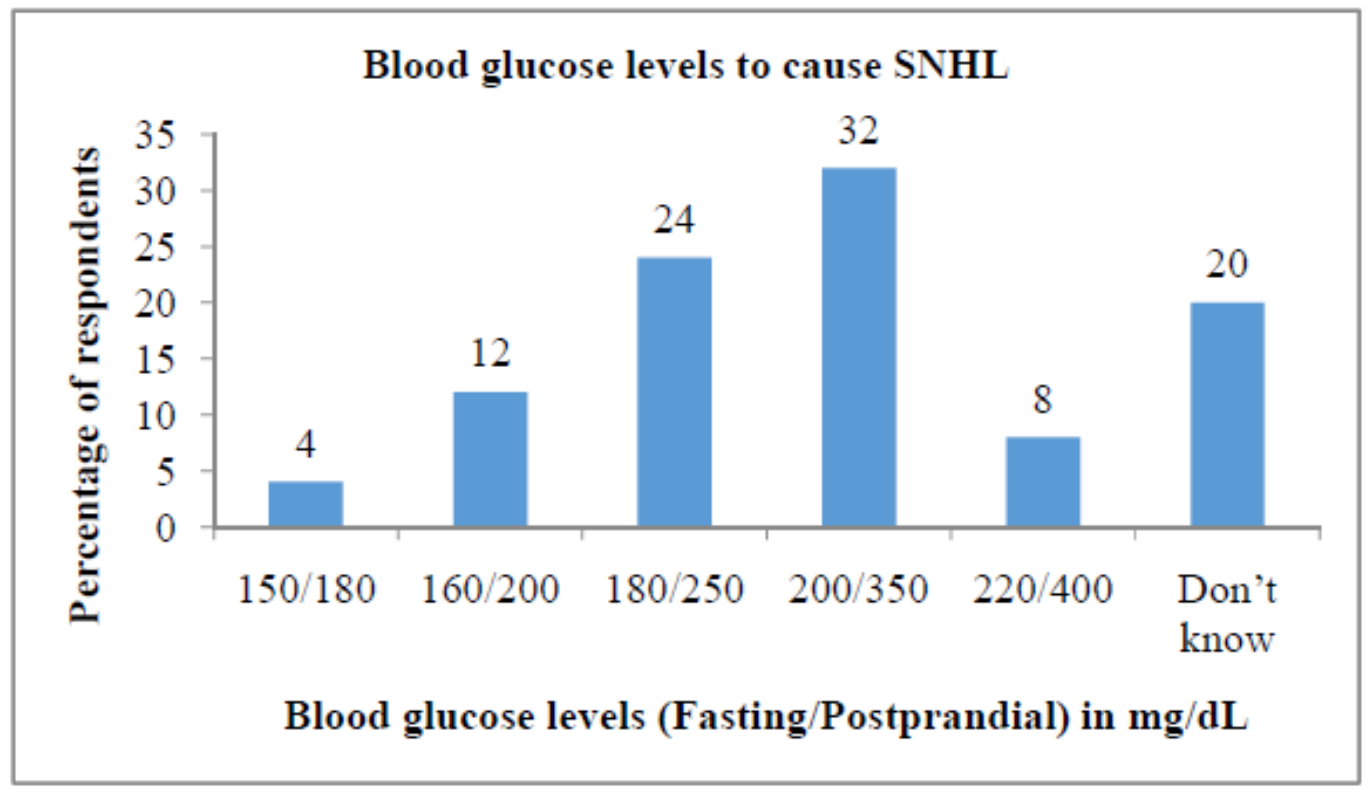

Figure 6: Blood glucose levels to cause SNHL

Duration of diabetes is also important to cause SNHL. Results indicate that diabetes from around 8 years duration can lead to SNHL. Results are shown graphically in figure 7.

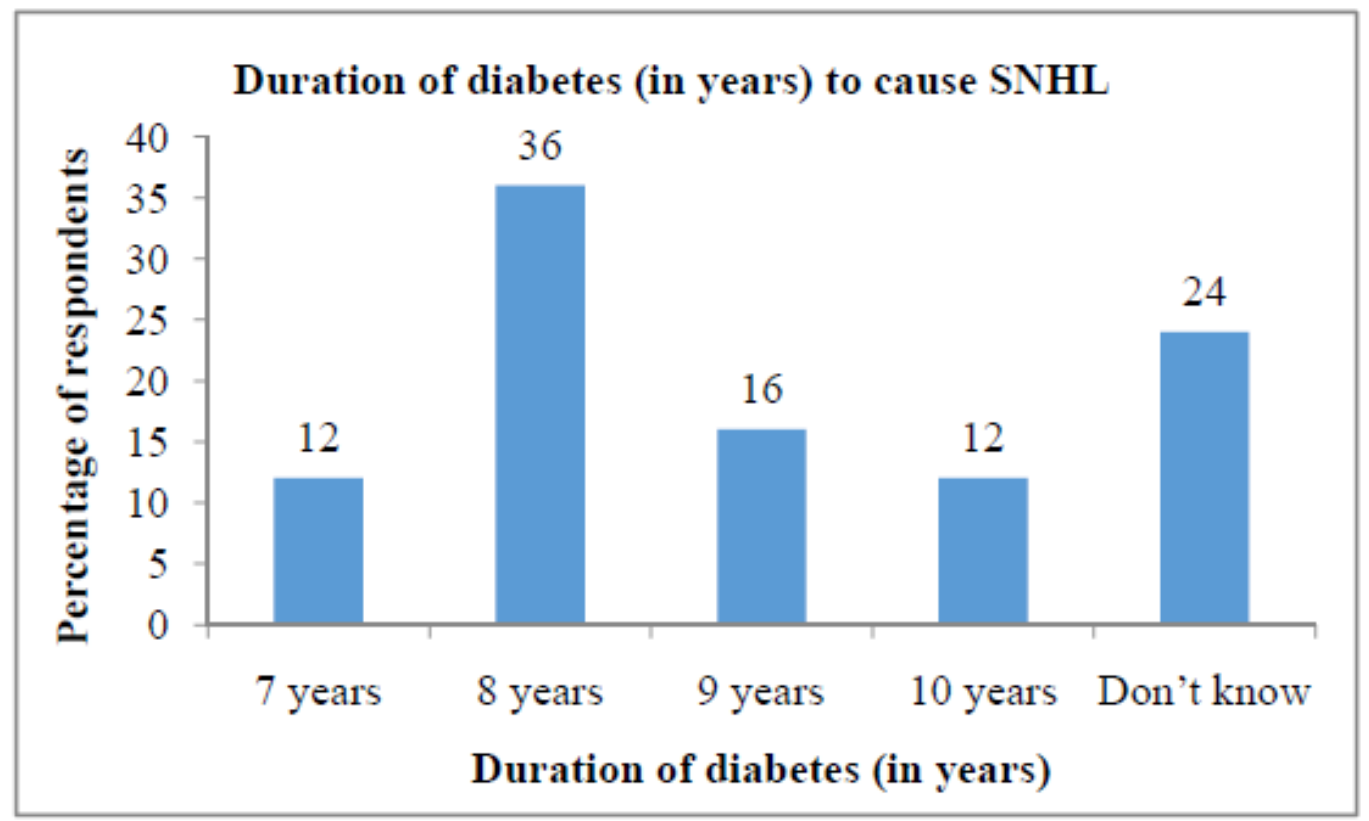

Figure 7: Duration of diabetes (in years) to cause SNHL

Insulin or oral hypoglycaemic drugs are prescribed for diabetes. Professionals were asked that whether taking insulin reduces sensorineural hearing loss as compared to taking oral hypoglycaemic drugs. Thirty six percent physicians answered that insulin reduces SNHL whereas 64\% do not know about any kind of such relationship. 
Obesity and high body mass index (BMI) are well known causes of diabetes. Hence, physicians were asked about relationship of obesity and high BMI with SNHL. 88\% of physicians and audiologists responded that there exists relationship of obesity and high with SNHL. Only twelve percent physicians think that there exists no relation between these factors.

Question was asked about relationship of high levels of lipids with causation of SNHL. Results showed that 52\% physicians think that high levels of triglycerides and $20 \%$ of physicians think that high levels of LDL cause SNHL.

\section{Discussion}

Both diabetes and hypertension are one of prevalent disorders in world and have their effect on other systems of body. Present study was to investigate relationship and frequency of sensorineural hearing loss (SNHL) due to previously diagnosed diabetes mellitus and/or hypertension in adult population of Mumbai metropolitan region.

\section{Patient factors}

Although response rate of $51.02 \%$ seems very low, possible reasons behind this might be recording of interview using Sony Walkman NW Z-B163F, non-familiarity with methodology of semi-structured interview or fear of opinions due to recording, etc. The reason explained by one of MD (Medicine) as we generally do not look after cases of hearing loss. However, during entire conduction of study, co-operation of audiologists and MS (ENT) physicians was encouraging. Many of them said there is need to conduct such bridging studies as people and even physicians take hearing loss conditions very lightly. Studies have revealed that more experience provide expertise to physicians in particular area. Experience of physicians is also important as it has capacity to provide right answers through their clinical judgement (Akl, Khairy, Abdel-Aal, Deghedi \& Amer 2006, Kim, Park H. G., Park E. C., \& Park K. 2011). During this study responses were obtained from physicians of experience 7 years to 43 years.

Particularly, audiologists and ENT surgeons look after large number of cases due to their expertise in hearing conditions. However, MBBS (being family physicians or general practitioners) and MD (Medicine) (Diabetologist/Cardiologist) look after less number of cases comparatively. Out of these diagnosed cases of SNHL by ENT surgeon, cases of diabetes were $71.43 \%$; cases of hypertension $39.29 \%$ and both conditions $53.57 \%$. This highlights higher prevalence of diabetes and hypertension in India. Studies by Friedman et al (1975) and Kakarlapudi et al. (2003) suggest that incidence of hearing loss in diabetic patients is around 30 percent to 95 percent. Findings of current study were in similar range. High frequencies of diabetes and hypertension in Indian population were earlier suggested by SITE study (Mozaffari, Tajik, Ariaei, Ali-Ehayaii \& Behnam 2010). According to many studies which have highlighted relationship of age and sensorineural hearing loss (Friedman, Schulman \& weiss 1975, Kakarlapudi, Sawyer \& Staecker 2003) occurrence of hearing loss increases after age 65. Similarly, report by Shield states that in UK, prevalence of hearing loss increases particularly in age groups 61-70 and 71-80 (Shield 2006).In present study, high frequencies of SNHL were found in age group of 50-60 (52\%). However, frequencies in age group 60-70 were 8\%. This seems contradictory with earlier studies. However, it must be noted that earlier studies (Shield 2006, Gates, Cooper \& Kannel 1990) were done in US, Europe and UK and no data from India was available. Therefore, there is necessity to conduct large studies to evaluate prevalence of hearing loss in India. Another reason might be high occurrence of diabetes and hypertension in Indian population ((Mozaffari, Tajik, Ariaei, Ali-Ehayaii \& Behnam 2010) which might pose to early age of hearing loss in Indian population. 
South American Journal of Clinical Research

Special Edition 2016

In UK, men were more prone to have hearing loss in age above 40. However, prevalence in women was less. In India, study by Rajendran et al. (2011) suggests that there is no gender difference in causation of sensorineural hearing loss. Findings of present study indicate that $56 \%$ of physicians said there is no discrimination of gender and $40 \%$ said hearing loss was more prevalent in men. There was mixed opinion (44\% both opinions saying no discrimination among all job descriptions and for factory workers) amongst physicians regarding working style or job descriptions of patients who had SNHL. However, studies indicate that workers which are working in industrial environment or noisy places were more prone to have SNHL.

\section{Physician's recommendations for hearing loss}

Semi-structured interviews of physicians suggest that sensorineural hearing loss occurs in both ears i.e. bilateral (76\%). Results of clinical trial done in 120 patients in India suggest that SNHL was bilateral in most patients (Rajendran, Anandhalakshmi \& Rao 2011) whereas study in South Korea showed that SNHL was unilateral. Pure tone audiometry was most preferred (72\%) by physicians and audiologists. Along with this tympanometry or impedance test was also referred by $28 \%$ physicians. These tests help in providing estimation of degree of hearing loss.

Considering close association of diabetes and blood pressure with SNHL, it is necessary to recommend patients of these conditions to have audiometric test at least once a year. Around 52\% of physicians and audiologists were aware of this fact and recommended audiometric tests. However, there are no such studies which signify importance of audiometric tests in diabetes and/or hypertension. There are no studies conducted in order to determine duration of diabetes and/or hypertension for conduction of audiometric tests. However, many physicians answered it could be 7 to 10 years. Majority of physicians (68\%) recommended that audiometric test should be conducted once a year so as to ensure normal hearing of patient of diabetes and/or hypertension.

Family and medical history of conditions play crucial role in diagnosis of disease conditions. Findings of McMahan CM et al (2008) suggests strong relationship between maternal history and hearing loss. Particularly in case of diabetics, maternal family history plays significant role in causation of deafness in North Americans. Results of current study showed close relationship of family history with sensorineural hearing loss as per $84 \%$ of physicians. In case medical history of disease, results of present study suggested that all ENT surgeons and audiologists ask patients about history of diabetes and/or hypertension while conduction of audiometric tests.

Inner ear pathology is useful in determining underlying cause of sensorineural hearing loss as it is used to investigate structural and pathological changes in inner ear. Based on this, line of treatment can be initiated. This study showed that referral for inner ear pathology was advised by only $20 \%$ of physicians.

\section{Hearing related conditions}

Semi-structured interviews suggested drug therapy as first choice option for sensorineural hearing loss and hearing aid as second preferred choice. Drug therapy includes use of steroids, vasodilators, diuretics and use of low salt diets with controlled intake of sugar (Patient.co.uk, Nagaoka, Anjos, Takata, Chaim, Barros \& Penido 2010). Therefore, findings of study were in accordance with recommendations of "Action on Hearing Loss, UK” (Patient.co.uk).

The choice of hearing aid depends totally on economical condition of patient as well as degree of severity of hearing loss. Completely in canal (CIC) type or in the canal (ITC) type of hearing aids were of no use if hearing impairment is profound. The age of patient is also another contributing factor for this. If age of patient is 
greater than 60 and patient has dexterity problems; then audiologists generally refer pocket model or behind the ear (BTE) type of hearing aid. Results of our study indicated that behind the ear type of hearing aid was mostly preferred by patients (60\% respondents). Govt. of India has National Program for Prevention and Control of Deafness (NPPCD) wherein hearing aids are provided and amount is reimbursed by governmental authority (Ministry of health and family welfare, Govt. of India). Particularly government provides reimbursement of 7000 INR for analogue BTE and 20,000 INR for digital BTE (Ministry of health and family welfare, Govt. of India). However, according to opinion of physicians, very few patients were aware of this scheme and avail this facility. Therefore, there is need to promote this program effectively by government. Even this program should be promoted in private clinics and hospitals so that every person with hearing impairment will get benefitted. Whenever, hearing impairment is profound, hearing aids are of no use. In such circumstances cochlear implants or bone conduction implants are necessary. Indian NPPCD program does not covers these implant procedures. However, there is need to devise such policy for implant procedures. In UK, these procedures are covered under NHS (Patient.co.uk).

Occurrence of tinnitus in SNHL patients suffering from diabetes and hypertension was $68 \%$ and in SNHL patients with diabetes was 16\%. Even physicians suggest that there are $80 \%$ cases of subjective type of tinnitus. This fact also highlighted earlier indicating presence of unilateral and bilateral tinnitus. The presence of tinnitus could be attributed to damage to hair cells of cochlea leading partial loss of inhibitory functions of associated neurons causing firing of them even when no sound is present. Opinion of one male ENT surgeon with 29 years of experience was found to be similar as that of mechanism mentioned above.

Dysfunction in vestibular system results in vertigo. All respondents said that there exists close association of vertigo and SNHL. This statement is in accordance with different studies. Persistent high frequency sensorineural hearing loss due to dysfunction in basal turn of cochlea and vestibule leads to vertigo. Study have showed this fact in clinical trial in 67 patients. Occlusive arterial disease of inner ear caused due to hypertension leads to vertigo. Therefore findings of current study indicate relation of vertigo with SNHL along with presence or hypertension and diabetes.

\section{Diabetes SNHL, hypertension}

Duration of hypertension: Report from US National Health and Nutrition Examination Survey from 1999 to 2002 suggest that cardiovascular risk factors (hypertension, smoking, and diabetes) are responsible for hearing loss ((Mozaffari, Tajik, Ariaei, Ali-Ehayaii \& Behnam 2010). Results of present study indicated that hypertension from 7 to 9 years (i.e. 4, 24 and 20\% of respondents) could lead to SNHL.

Range of blood pressure and underlying mechanism for SNHL: Whenever range of blood pressure was concerned, 40\% respondents said that 160/110 mmHg (systolic and diastolic) blood pressure was responsible to cause SNHL. The reason behind causation of SNHL due to hypertension is attributed to structural changes in blood supply of inner ear or inner ear haemorrhage leading to sudden or progressive hearing loss. Underlying pathological mechanisms might be increased blood viscosity, reduction in capillary blood flow, reduction in oxygen transport, hypoxia, and changes in ionic cell potentials. These cumulatively lead to hypertensive retinopathy (i.e. damage in end organ blood vessels). This in turn results in problems in hearing capacity (Kakarlapudi, Sayer \& Staecker 2003, Marchiori, Filbo \& Mastuo 2006). However, there is need of further investigation about duration of hypertension and its relation to severity of SNHL. 
South American Journal of Clinical Research

Special Edition 2016

Results of study indicate that all physicians did not have knowledge about effect of antihypertensive drugs on hearing loss. However, literature suggests that diuretics were more prone to cause tinnitus and hearing loss as compared to angiotensin II receptor blockers.

Insulin versus hypoglycaemic agents and its effect on SNHL: This was much debated amongst scientific community that taking insulin for control of glycaemia would reduce diabetes and in turn SNHL. Thirty six percent of respondents of semistructured interview suggested that insulin reduces causation and progression of SNHL and 64\% were unaware of any such kind of relationship. However, single blinded randomised controlled study by Celik et al. (1996) showed that neither insulin treatment nor oral hypoglycemics have effect on hearing thresholds. However, this study involved only 28 participants. Therefore, there is need to conduct randomised controlled trial involving more number of subjects of various ethnicities.

Blood glucose levels: Around 32\% of physicians said that glucose levels of fasting $200 \mathrm{mg} / \mathrm{dl}$ and postprandial $350 \mathrm{mg} / \mathrm{dl}$ can cause SNHL. Similar results were obtained earlier by Rajendran et al (2011) which showed that $73.3 \%$ of diabetic individuals have SNHL with levels of glucose fasting $181 \mathrm{mg} / \mathrm{dl}$ and postprandial $286 \mathrm{mg} / \mathrm{dl}$.

Duration of diabetes: Results of semi-structured interviews indicated that duration of diabetes from around 8 years could lead to SNHL. Similarly, in case control study, it was observed that diabetes was present since 5 to 15 years (2 cases of 5-10 years, 3 cases of 10-15 years). These results seem somewhat contrasting to results of earlier studies. Study by Rajendran et al. (2011) stated that duration of diabetes above or below 10 years including control of glycaemia of above or below 8 years had no effect on incidence of SNHL. It was also observed that increase in duration of diabetes up to 15 years, incidence of SNHL increased (Celik, Yalcin, Celebi, \& Ozturk 1996). One study indicated higher incidence of hearing loss in females whereas other indicated higher incidence in males. In present study physicians opined that males were more prone to SNHL than females. There is need for further investigation using randomised controlled clinical trial. Relationship of obesity, high BMI with hypertension and diabetes is well known. Physicians in present study also said same fact. 88\% of physicians opined that obesity and high BMI could cause diabetes and hypertension which in turn could lead to SNHL. Report by Shield (2006) state that obesity and high BMI were related to SNHL.

Results showed that $52 \%$ of physicians think high triglycerides and $20 \%$ physicians think high low density lipoprotein (LDL) levels were responsible factor for causation of SNHL. Results of this study were in accordance with earlier studies. The underlying mechanism behind causation of SNHL due to higher lipid levels was causation of spasm of spiralismodiolic artery and vestibulocochlear artery due to lipids (Oreskovi, Shejbal, Bicanic \& Kekic 2010, Swaminathan, Sambandam \& Bhaskaran 2011).

\section{Conclusions}

Sensorineural hearing loss is most commonly observed form of hearing loss which occurs due to damage to inner ear or nerve pathways (particularly in vestibulocochler nerve or cranial nerve VIII) or problems in central processing of brain. Diabetes and hypertension accounts for major healthcare problem in India. Literature search revealed that both diabetes and hypertension play important role in causation of sensorineural hearing loss. Therefore, there was need to evaluate relationship and frequency of diabetes and/or hypertension with sensorineural hearing loss.

Findings of study revealed that there exists close association of diabetes and/or hypertension with sensorineural hearing loss. It was found that out of all SNHL patients, there were $71.43 \%$ cases of diabetes; $39.29 \%$ cases of hypertension; and $53.57 \%$ of both diabetes and hypertension. SNHL was found to be more prevalent in 
age group 50-60 years with moderate degree. It was found that medical and family history of diabetes and/or hypertension plays important role in causation of SNHL. Behind the ear (BTE) type of hearing aid was most used in patients of SNHL. High LDL, BMI, blood pressure and glucose levels are risk factors for causation of SNHL. Tinnitus and vertigo also have close relationship with causation of SNHL.

There is need to conduct large scale multicentric randomised controlled clinical trial to investigate and confirm association of diabetes and/or hypertension with sensorineural hearing loss. Apart from this, awareness amongst public regarding hearing loss, usefulness of hearing aid, control of diabetes and/or hypertension is essential.

\section{References}

[1]. Agrawal, Y., Platz, E., A., Niparko, J., K. (2009). Risk Factors for Hearing Loss in US Adults: Data from the National Health and Nutrition Examination Survey, 1999 to 2002. Otology and Nutrology. 30, 139-145.

[2]. Akl, O., A., Khairy, A., E., Abdel-Aal, N., M., Deghedi, B., S., Amer, Z., F. (2006). Knowledge, attitude, practice and performance of family physicians concerning holistic management of hypertension. J Egypt Public Health Assoc, 81(5), 337-353.

[3]. Arogyadeep: Directory of health services. 3rd ed. Rotary Vikas Trust, Dombivli, India. 2013.

[4]. Bhattacharyya, A., K., Thaj, J. (2010) Investigation protocol for sensorineural hearing loss. Otorhino larungology Clinics: An international Journal, 2(2), 107-112.

[5]. Celik, O., Yalçin, S., Celebi, H., Oztürk, A. (1996). Hearing loss in insulin dependent diabetes mellitus. AurisNasus Larynx, 23, 127-132.

[6]. Clinical management of primary hypertension in adults. NICE Clinical Guideline 127. Available at: http://www.nice.org.uk/nicemedia/live/13561/56008/56008.pdf (Accessed on 11 December 2013).

[7]. Costa. O., A. (1967) Inner ear pathology in experimental diabetes. Laryngoscope, 77, 68-75.

[8]. Davies, M., B. Doing a successful research project: Using qualitative or quantitative methods. Palgrave Macmillan, Hampshire, UK. 2007.

[9]. Diabetes and high blood pressure. Patient.co.uk. Available at: http://www.patient.co.uk/health/diabetes-andhigh-blood-pressure (Accessed on 01 December 2013).

[10]. Diniz, T., H., Guida, H., L. (2009). Hearing loss in patients with diabetes mellitus. Braz J Otorhinolaryngol, 75(4), 573-578.

[11]. Duthey, B. (2013). Background paper 6.21 Hearing loss. Priority Medicines for Europe and the World "A Public Health Approach to Innovation". World Health Organisation (WHO) Available at: http://www.who.int/medicines/areas/priority_medicines/BP6_21Hearing.pdf (Accessed on 12 November 2013)

[12]. Esparza, C., M., Jauregui-Renaud, K., Morelos, C., M., C., Muhl, G., E., A., Mendez, M., N., Carilo, N., S., Bello, N., S., Cardenas, M. (2007). Systemic high blood pressure and inner ear dysfunction: a preliminary study. ClinOtolaryngol, 32, 173-178.

[13]. Friedman, S., A., Schulman, R., H., Weiss, S. (1975). Hearing and diabetic neuropathy. Archives of Internal Medicine, 135, 573-576.

[14]. Gates, G., A., Cooper, J., C., Kannel, W., B., et al. (1990). Hearing in the elderly. The Framinghamcohort, 1983-1985, Part 1, basic audiometric test results. Ear and Hearing, 11 (4), 247-256.

[15]. Joshi, S., R., Vadivale, M., Dalal, J., J., Das, A., K. (2011). The Screening India’s Twin Epidemic: study design and methodology (SITE-1). Indian J EndocrinolMetab, (Suppl4), S389- S394

[16]. Kakarlapudi, V., Sawyer, R., Staecker, H. (2003). The effect of diabetes on sensorineural hearing loss. OtolNeurotol, 24(3), 382-386.

[17]. Kim, M., H., Park, H., G., Park, E., C., Park, K. (2011). Attitude and knowledge of physicians about cancer pain management: young doctors of South Korea in their early career. Jpn. J. Clin. Oncol, 41(6)783-791.

[18]. Latoche, J. R., Neely, H., R., Noben-Trauth, K. (2011). Polygenic inheritance of sensorineural hearing loss (Snhl-2,3 and 4) and organ of corti patterning defect in ALR /Ltj mouse strain. Hearing Research, 275, 150-159.

[19]. Makishima, K., Tanaka, A., K. (1971). Pathological changes of the inner ear and central auditory pathway in diabetes. Ann Otol Rhinol Laryngol, 80(2), 218-228.

[20]. Marchiori, LLDM, Filbo, EDAR, Mastuo, T. (2006). Hypertension as factor associated with hearing loss. Rev Bras Otolaringol, 72, 533-540. 


\section{South American Journal of Clinical Research Special Edition 2016}

[21]. McCormick, J., G., Harris, D., T., Hartley, C., B., Lassiter, B., H. (1982). Spontaneous genetic hypertension in the rat and its relationship to reduced ac cochlear potentials: Implications for preservation of human hearing. Proc. Natl. Acad. Sci. USA, 79, 2668-2672.

[22]. McMahon, C., M., Kifley, A., Rochtchina, E., Newall, P., Mitchell, P. (2008). The contribution of family history to hearing loss in an older population. Ear Hear, 29, 578-584.

[23]. Mondell, MFCG, Lopes, A., C. (2009). Relation between arterial hypertension and hearing loss. Int. Arch. Otorhinolaryngol, 13, 63-68.

[24]. Mozaffari. M., Tajik, A., Ariaei, N., Ali-Ehayaii, F., Behnam, H. (2010) Diabetes mellitus and sensorineural hearing loss among non-elderly people. Eastern Mediterranean Health Journal, 16(9), 947-952.

[25]. Nagaoka, J., Anjos, MFd, Takata, T., T., Chaim, R., M., Barros, F., Penido, NdO. (2010). Idiopathic sudden sensorineural hearing loss: evolution in presence of hypertension, diabetes mellitus and dyslipidemia. Braz $J$ Otorhinolaryngol, 76, 363-369.

[26]. Nishio, N., Teranishi, M., Uchida, Y., Sugiura, S., Ando, F., Shimokata, H., et al. (2012). Contribution of complement factor HY 402H polymorphism to sudden sensorineural hearing loss risk and possible interaction with diabetes. Gene, 499, 226-230.

[27]. Oreskovi, Z., Shejbal, D., Bicanic, G., Kekic, B. (2010). Influence of lipoproteins and fibrinogen on pathogenesis of sudden sensorineural hearing loss. The Journal of Laryngology \& Otology, 1-4. doi:10.1017/S0022215110002252.

[28]. Project Deaf India Available at: http://projectdeafindia.org/news/hope-for-hearing-impairedpeople-in-india/ (Accessed on: 12 June 2013)

[29]. Rajendran, S., Anandhalakshmi, M., B., Rao, V. (2011). Evaluation of incidence of sensorineural hearing loss in patients with type 2 diabetes mellitus. Int J Biol Med Res, 2(4), 982-987.

[30]. Revision of rates and guidelines for reimbursement of expenses on purchase of hearing aids under CS(MA) rules, 1944 and CGH S-regarding. Ministry of health and family welfare, Govt. of India. S14025/10/2002/MS. 21 March 2012.

[31]. Shield, B. (2006). Evaluation of social and economic costs of hearing impairment. Hear-It. Available at: http://www.hear-it.org/multimedia/Hear_It_Report_October_2006.pdf (Accessed on 12 November 2013

[32]. Smith, T., L., Raynor, E., Prazma, J., Buenting, J., E., Pillsbury, H., C. (1995) Insulin dependent diabetic microangiopathy in the inner ear. J Laryngol Otol.105, 236-240.

[33]. Sui, L., I., Shusheng, G., Yanzen, Y., Qingsong, Yu. (2003). Effect of Hypertension on Hearing Function, LDH and ChE of the Cochlea in Older Rats. Journal of Huazhong University of Science and Technology [Med Sci]. 23, 306-309.

[34]. Swaminathan, A., Sambandam, R., Mythili, Bhaskaran. (2011). Evaluation of auditory effects of hyperlipidemia and diabetes mellitus by using audiometry. Journal of clinical and diagnostic research, 5(8), 15281532.

[35]. Tan, T., Y., Rahmat, O., Prepageran, N., Fauzi, A., Noran, N., H., Raman, R. (2009). Hypertensive retinopathy and sensorineural hearing loss. Ind J Otolaryngol Head Neck Surg. 61, 275-279.

[36]. Taylor. I., G., Irwin, J. (1978). Some audiological aspects of diabetes mellitus. J Laryngol Otol, 92(2), 99113.

[37]. Viswanathan, M., Seedat, Y., K., Pradeepa, R. (2013). The Rising Burden of Diabetes and Hypertension in Southeast Asian and African Regions: Need for Effective Strategies for Prevention and Control in Primary Health Care Settings. Int J Hypertension, 2013: Article ID409083, doi:10.1155/2013/409083.

[38]. Wallhagen, M., I., Strawbridge, W., J., Cohen, R., D., Kaplan, G., A. (1997). An increasing prevalence of hearing impairment and associated risk factors over three decades of the Alameda County study. American Journal of Public Health, 87(3), 440-442. 